\title{
On a Converse theorem for Finite-time Lyapunov Functions to Estimate Domains of Attraction
}

\author{
Ayush Pandey Aaron D. Ames
}

\begin{abstract}
The main result of the paper is a new converse theorem for finite-time Lyapunov functions. We show the existence of a finite-time Lyapunov function for an autonomous continuoustime nonlinear dynamical system if the origin of the system is asymptotically stable. Our proof extends the recent results in finite-time Lyapunov function theory by providing an alternative converse proof for the existence of finite-time Lyapunov functions. In particular, we show that given asymptotic stability of the origin, the linearized dynamics satisfy global finitetime Lyapunov function conditions hence proving the converse theorem. Using our results, we present a consolidated theory for using and constructing Lyapunov functions to certify system stability properties. We also propose a constructive algorithm to efficiently compute non-conservative estimates of the domain of attraction for nonlinear dynamical systems.
\end{abstract}

\section{INTRODUCTION}

The classical way of showing existence of Lyapunov functions is due to Massera [1], where integration of system solutions are used to construct a Lyapunov function (LF) that certifies the stability properties of the equilibrium point. Given a certain condition on convergence of the system trajectories to the equilibrium point, we can show the existence of a LF to prove the converse Lyapunov theorem. The Massera-type LF is constructed by a semi-definite integral of an appropriately chosen positive definite function of the norm of the system solutions. However, there is no clear choice or a constructive method to find this function so that the integral converges and gives a valid LF. Hence, for general nonlinear system dynamics it is difficult to explicitly construct a LF [2]. As a result, there has been continued interest in synthesizing LFs and developing converse results to the Lyapunov stability theory. The reader is referred to the papers [3] and [4] for a good survey of existing converse results.

It is important to have converse LF results that can be used to constructively compute LFs. Such results would not only result in efficient computations of stability properties and design of controllers but also for computing an estimate of the domain of attraction of an equilibrium that has many engineering applications. However, the existing constructive approaches face various challenges such as - relying on complex candidate LFs that are developed for polynomial [5], rational [6], or quadratic [7] systems; involving state-space partitions or transformations that often face scalability

The authors are with the Department of Control and Dynamical Systems, California Institute of Technology, Pasadena, CA. Email: apandey@caltech.edu, ames@ caltech.edu issues [8]; formulating the problem as a complex and large optimization problem [9] that is computationally inefficient even for simple system dynamics. As a step towards an analytical approach to address these issues, it has been shown recently in [10] that finite-time Lyapunov functions (FTLF) could be used to give a computationally efficient constructive approach to compute LFs.

A FTLF is a relaxation of the classical LFs originally proposed in [11]. FTLFs have been shown to be particularly important for stability theory of discrete-time [12]-[14] and time-variant nonlinear systems [11], [15]. The decrescent condition in the classical Lyapunov theory can be viewed as an infinitesimal version of the decrescent condition in FTLF theory. The FTLF theory can be used to provide constructive algorithms to compute a classical LF efficiently. Due to these advantages and relaxations, FTLFs have been used for various applications such as domain of attraction estimate [10] and simpler stability certificates [16] for complicated systems.

In this paper, we build upon and extend the recent results in [10] to prove the existence of a FTLF given asymptotic stability of the equilibrium. In [10], the converse theorem is directly proved for the nonlinear system using FTLF properties and a Massera-type construction. We take a different approach by proving the existence of a global FTLF for the linearization of a nonlinear system to prove the same converse theorem. We highlight the implications of this proof by presenting a consolidated theory that connects our results with the results in [10] to give a simplified approach to constructively compute LFs. We also develop a numerical approximation based algorithm to give a computational implementation that can be used to compute the domain of attraction estimates for general nonlinear systems.

The paper is organized into five sections. We will discuss some preliminary topics in the next section that are important to prove the main results in Section III. Using these results, we will present a simple computational procedure to estimate the domain of attraction for general continuous-time nonlinear dynamical system in Section IV. Finally, in Section V, we will consider a biologically relevant system of a bistable toggle switch and use our results to estimate the domains of attraction for its asymptotically stable equilibrium points. 


\section{Preliminaries}

We consider an autonomous nonlinear dynamical system described by ordinary differential equations where $x \in$ $E \subset \mathbb{R}^{n}$ denotes the system state vector, where $E$ is an open connected subset of $\mathbb{R}^{n}$ that contains the origin. The dynamics are given by

$$
\dot{x}=f(x)
$$

for a locally Lipschitz function $f: E \subset \mathbb{R}^{n} \rightarrow \mathbb{R}^{n}$ and initial conditions $x(0) \in E$. Without loss of generality, we assume that the system has an equilibrium point at the origin denoted by $x^{*}=0$, i.e. $f\left(x^{*}\right)=0$. The local Lipschitz continuity of $f$ gives us that the solution $x(t)$ exists and is unique for a finite time interval. We further assume that the solutions exist for all $t \geq 0$. See [17, Corollary 2.5] for the sufficient smoothness conditions that are needed on $f$ for this assumption to hold. We also have that the solutions depend continuously on the initial condition $x(0)$, i.e., $x(t)$ is a continuous function of $x(0)$ over any finite time interval. See [18, Theorem 3.4] for a proof and related discussion. We now give some notation and definitions that will be important in deriving the results in this paper.

Definition 1. The logarithmic norm [19] of a matrix $A$ is a real valued functional given by

$$
\mu(A)=\lim _{h \rightarrow 0^{+}} \frac{\|I+h A\|-1}{h}
$$

where $\|$.$\| is any vector norm.$

Remark. An important property of the logarithmic norm that holds for all $t \geq 0$ is given below:

$$
\left\|e^{A t}\right\| \leq e^{\mu(A) t} .
$$

Recall that a set $S \subseteq \mathbb{R}^{n}$ is called a proper set if it is compact and contains the equilibrium point in its interior, i.e., $x^{*}=0 \in \operatorname{int}(S)$.

Definition 2. A proper set $S \subseteq \mathbb{R}^{n}$ is called an invariant set with respect to the solution flow $\varphi_{t}$ of system (1) if $\varphi_{t}(S) \subset S$, i.e., $\varphi_{t}(x) \in S$ for all $x(t) \in S$ and all $t \geq 0$. Further, for a given positive, real scalar $d$ and a proper set $S \subset \mathbb{R}^{n}$, if $\varphi_{t+d}(x) \in S$ for all $x(t) \in S$ and all $t \geq 0$, then $S$ is called a $d$-invariant set for the system (1).

Recall that a continuous function $\alpha:[0, a) \rightarrow[0, \infty)$, is of class $\mathcal{K}$ if it is strictly increasing and $\alpha(0)=0$. Similarly, a continuous function $\alpha:[0, a) \rightarrow[0, \infty)$ is of class $\mathcal{K}_{\infty}$ if it is strictly increasing, $\alpha(0)=0$, and $\lim _{r \rightarrow \infty} \alpha(r)=\infty$. Further, a continuous function $\beta:[0, a) \times[0, \infty) \rightarrow[0, \infty)$ is of class $\mathcal{K} \mathcal{L}$ if, for each fixed $t \in[0, \infty), \beta(s, t)$ is of class $\mathcal{K}$ with respect to $s$ and, for each fixed $s \in[0, a)$, we have

$$
\lim _{t \rightarrow \infty} \beta(s, t)=0 .
$$

Lemma 1. An equilibrium point $x^{*}=0$ of $\dot{x}=f(x)$ is $\mathcal{K} \mathcal{L}$ stable if there exists an $a \in \mathbb{R}_{>0}$ and a class $\mathcal{K} \mathcal{L}$ function $\beta:[0, a) \times[0, \infty) \rightarrow[0, \infty)$ such that

$$
\|x(0)\| \leq a \quad \Rightarrow \quad\|x(t)\| \leq \beta(\|x(0)\|, t) .
$$

This notion of stability leads to the "modern" statement of Lyapunov theorem in terms of class $\mathcal{K}$ and $\mathcal{K} \mathcal{L}$ functions.

Theorem 1. For the system in (1) and a continuously differentiable function $V: E \rightarrow \mathbb{R}^{n}$, if the following conditions are satisfied for all $x \in E$ :

$$
\begin{gathered}
\alpha_{1}(\|x\|) \leq V(x) \leq \alpha_{2}(\|x\|) \\
\dot{V}(x) \leq-\alpha_{3}(\|x\|)
\end{gathered}
$$

for $\alpha_{i} \in \mathcal{K}_{\infty}, i=1,2,3$, then $x^{*}=0$ is $\mathcal{K} \mathcal{L}$-stable. Moreover,

$$
\|x(t)\| \leq \alpha_{1}^{-1} \beta\left(\alpha_{2}(\|x(0)\|, t)\right)
$$

for all $t \geq 0$ and $x(0) \in E$, where $\beta \in \mathcal{K} \mathcal{L}$ is the solution to the following initial value problem:

$$
\dot{y}=-\alpha_{3}\left(\alpha_{2}^{-1}(y)\right), \quad y(0)=V(x(0)) .
$$

The converse of this results is given next.

Theorem 2. For the system in (1) with $E=B_{r}(0)$, a ball of radius $r>0$ around the origin, if the equilibrium point $x^{*}=0 \in E$ is $\mathcal{K} \mathcal{L}$-stable for all $x(0) \in E$, i.e.,

$$
\|x(t)\| \leq \beta(\|x(0)\|, t)
$$

then, there exist class $\mathcal{K}$ functions $\alpha_{i}, i=1,2,3, r_{0}>0$, and a $L F V: B_{r_{0}}(0) \rightarrow \mathbb{R}$ satisfying equations (5) and (6).

The proofs of these two theorems can be found in any standard textbook on Lyapunov theory [17], [18] along with other versions of the converse Lyapunov theorem. From [20], the following lemma will be integral for our results in this paper:

Lemma 2. For each class $\mathcal{K} \mathcal{L}$-function $\beta$ and each number $\lambda \in \mathbb{R}_{\geq 0}$, there exists a $\phi \in \mathcal{K}_{\infty}$, such that

$$
\beta(s, t) \leq \phi(s) e^{-\lambda t}
$$

Finally, we define a finite-time Lyapunov function as given in [10] before proceeding to the main results that prove system stability properties using FTLFs.

Definition 3. A continuous function $V: \mathbb{R}^{n} \rightarrow \mathbb{R}_{\geq 0}$ is called a finite-time Lyapunov function if for a real scalar $d>0$ for which the proper set $S \subset \mathbb{R}^{n}$ is $d$-invariant we have that

$$
\begin{gathered}
\alpha_{1}(\|x\|) \leq V(x) \leq \alpha_{2}(\|x\|) \\
V(x(t+d))-V(x(t)) \leq-\gamma(\|x\|)
\end{gathered}
$$

for all $x(t)$ with $x(0) \in S$ for all $t \geq 0$ where $\alpha_{1}, \alpha_{2} \in \mathcal{K}_{\infty}$ and $\gamma \in \mathcal{K}$.

As given in [10], in order for equation (12) to be well defined, it is assumed that there exists no finite escape time in the interval $[0, d]$ for all $t \geq 0$. 


\section{Results}

We will present the main results of this paper in this section. To build up to these results, we will need the following lemma,

Lemma 3 (Lemma 2.1 [10]). The FTLF decrease condition in equation (12) is equivalent to

$$
V(x(t+d))-\rho(V(x(t)) \leq 0
$$

for all $t \geq 0$ and for all $x(t)$ with $x(0) \in S$, where $\rho$ : $\mathbb{R}_{\geq 0} \rightarrow \mathbb{R}_{\geq 0}$ is a continuous positive definite function that is less than the identity function, $\rho<i d$ and $\rho(0)=0$.

This equivalent condition will be important in our converse results later. We will also need the following definition:

Definition 4. A function $V(x)=\|x\|$ is a global FTLF for the linearized system $\dot{x}=A x$ if we have

$$
\begin{aligned}
& \text { - }\left\|e^{A d}\right\|<1 \\
& \text { - } e^{d \mu(A)}-1=-\zeta \text {, for a } \zeta \in \mathbb{R}_{>0} \text { and } d>0 \text {. }
\end{aligned}
$$

Before going into our results, we summarize the results of [10] next. The result in [10, Theorem 2.1] shows that if there exists a FTLF as given in Definition 3 satisfying (11) and (12) and a proper $d$-invariant set $\mathrm{S}$ for system (1), then the equilibrium point $x^{*}=0$ is $\mathcal{K} \mathcal{L}$-stable in $S$. We further have the following from [10, Theorem 4.1].

Theorem 3. If $V(x)=\|x\|$ is a global FTLF for the linearization $\dot{x}=A x$ of the system (1), then the following statements hold

- There exists a d-invariant set $S$ for which $V(x)$ is a FTLF for the system (1).

- There exists a set $\mathcal{A} \subseteq S$ for which

$$
W(x)=\int_{0}^{d} V(x+\tau f(x)) d \tau
$$

is a LF for system (1).

Building on these results, we give the main result of this paper in the following theorem that shows that the $\mathcal{K} \mathcal{L}$ stability of the equilibrium point for the system (1) implies global FTLF properties hold for the linearization.

Theorem 4. If the equilibrium point $x^{*}=0$ for (1) is $\mathcal{K} \mathcal{L}$ stable in some invariant proper subset of $\mathbb{R}^{n}, S$, then there exists a $L F V(x)=\|x\|$ and $a d>0$ that certifies the global FTLF properties for $\dot{x}=A x$. Therefore, there exists a d-invariant set $S$ for which $V(x)=\|x\|$ is a FTLF for system (1).

Before proving this result, we review standard constructions on linearizing a nonlinear system [18]. For the system in (1), we have $\dot{x}=f(x)$ where $f: E \rightarrow \mathbb{R}^{n}$ with $E \subset \mathbb{R}^{n}$ and $x^{*}=0 \in E$ is an equilibrium point. Consider a ball $B_{r}(0)=$ $\{x \in E:\|x\|<r\}$ and define the function $z_{i}(s):=f_{i}(s x)$ for $x \in B_{r}(0)$ and $s \in[0,1]$, where $f_{i}$ is the $i^{t h}$ component of the system dynamics $f$. Using the mean value theorem, we can write

$$
f_{i}(x)=z_{i}(1)-z_{i}(0)=\int_{0}^{1} \frac{d}{d t}\left(z_{i}(\tau)\right) d \tau .
$$

Using chain rule, we get

$$
f_{i}(x)=\left(\int_{0}^{1} \frac{d f_{i}(\tau x)}{d x} d \tau\right) x \triangleq \int_{0}^{1} D f_{i}(\tau x) x d \tau
$$

Since we need to prove global FTLF properties of the linearized system, we introduce the linearized dynamics ( $A=D f(0)$ ) by writing

$$
\dot{x}=f(x)=D f(0) x+\left(\left(\int_{0}^{1} D f(\tau x) d \tau\right)-D f(0)\right) x .
$$

Now, define the second term in equation (15) as

$$
g(x):=\left(\left(\int_{0}^{1} D f(\tau x) d \tau\right)-D f(0)\right) x
$$

to write $\dot{x}=A x+g(x)$ where $A=D f(0)$. We now have the necessary background in which to prove the main result.

Proof. To solve for $x(d)$ we multiply $e^{-A d}$ to the decomposition $\dot{x}=A x+g(x)$ and get,

$$
e^{-A d} \dot{x}(d)-e^{A d} A x(d)=e^{-A d} g(x(d)) .
$$

Using chain rule and integrating with appropriate limits, we obtain,

$$
x(d)=e^{A d} x(0)+\int_{0}^{d} e^{(d-\tau) A} g(x(\tau)) d \tau .
$$

Taking any norm and using the norm properties, we get an upper bound

$$
\|x(d)\| \leq\left\|e^{A d}\right\|\|x(0)\|+\int_{0}^{d}\left\|e^{(d-\tau) A} g(x(\tau))\right\| d \tau .
$$

For the remainder term defined in equation (16) we can write,

$$
\begin{aligned}
\lim _{x \rightarrow 0} \frac{\|g(x)\|}{\|x\|} & \leq\left\|\left(\int_{0}^{1} D f(\tau x) d \tau\right)-D f(0)\right\| \\
& =\left\|\lim _{x \rightarrow 0}\left(\int_{0}^{1} D f(\tau x) d \tau\right)-D f(0)\right\|=0
\end{aligned}
$$

Hence, for any $N>0$, there exists an $r>0$ such that $\|g(x)\| \leq N\|x\|$ for $x \in B_{r}(0)$. Using this bound and the Gronwall-Bellman lemma [21, p. 56] we get

$$
\begin{aligned}
\| x(d)) \| & \leq\left\|e^{A d}\right\|\|x(0)\| e^{N \int_{0}^{d}\left\|e^{(d-\tau) A}\right\|} d \tau \\
& \leq\left\|e^{A d}\right\|\|x(0)\| e^{N \int_{0}^{d} e^{(d-\tau) \mu(A)}} d \tau \\
& \leq\|x(0)\| e^{d \mu(A)+N \frac{e^{d \mu(A)}-1}{\mu A}}
\end{aligned}
$$

where we used the property of log-norm given in equation (3) to obtain the last inequality. Choose $V(x)=\|x\|$ to write,

$$
V(x(d)) \leq e^{d \mu(A)+N \frac{e^{d \mu(A)}-1}{\mu A}} V(x(0)) .
$$


Define $\rho:=e^{d \mu(A)+N \frac{e^{d \mu(A)}-1}{\mu A}}$ to write

$$
V(x(d))-\rho(V(x(0))) \leq 0,
$$

clearly the function $\rho$ is a continuous positive definite function. From the theorem statement, we know that the system trajectories are $\mathcal{K} \mathcal{L}$-stable, hence we have,

$$
\|x(d)\| \leq \beta(\|x(0)\|, d) .
$$

Using Lemma 2 with some $\lambda \in \mathbb{R}_{\geq 0}$ we obtain

$$
0 \leq V(x(d)) \leq \beta(V(x(0)), d) \leq \phi(V(x(0))) e^{-\lambda d} .
$$

Define a function $\eta(s)=\phi(s) e^{-\lambda d}$ and simplify further to write

$$
V(x(d))-\eta(V(x(0)) \leq 0
$$

Observe that $\eta<i d$ since a $\lambda>0$ can always be chosen such that there is a function $\phi$ that gives $\eta(s)=\phi(s) e^{-\lambda d}<$ $i d$. Comparing with equation (21), we have that

$$
d \mu(A)+N \frac{e^{d \mu(A)}-1}{\mu(A)}<0
$$

to ensure that $\rho<i d$. Consider the case when $\mu(A)>0$, on simplifying the expression in equation (22) we have that

$$
e^{d \mu(A)}-1<-\frac{d \mu(A)^{2}}{N},
$$

which is a contradiction. Hence, we have that $\mu(A)<0$. Thus, on using the log-norm property from equation (3), we get

$$
\left\|e^{A d}\right\| \leq e^{d \mu(A)}<1
$$

On simplifying the expression in equation (22) we get

$$
0<-\left(e^{d \mu(A)}-1\right)<\frac{d \mu(A)^{2}}{N}
$$

hence there exists a $\zeta \in \mathbb{R}_{>0}$ such that

$$
e^{d \mu(A)}-1=-\zeta \text {. }
$$

So, we have that $V(x)$ is a global FTLF for the linearization. To complete the proof, recall from Theorem 3 that global FTLF for the linearization implies existence of FTLF for the nonlinear system (1). Hence, we have shown that given $\mathcal{K} \mathcal{L}$ stability for an equilibrium point, there exists a global FTLF for the linearization and hence a FTLF for the nonlinear system, $V(x)=\|x\|$.

Remark. Note that the converse result that $\mathcal{K} \mathcal{L}$-stability implies existence of a FTLF has been shown in [10] in Theorem 2.2 and Lemma 2.2 therein. Although our result essentially proves the same result but the proof is different since we first prove that $\mathcal{K} \mathcal{L}$-stability implies global FTLF properties hold for the linearization which is then utilized to prove the converse result. The implications of this proof approach will be discussed further as we connect our results with the results in [10] to give a consolidated theorem next. The approach would also simplify the computational procedure as discussed in the next section.
In classical Lyapunov theory, we have that the following statements are equivalent (given all the assumptions made for system (1) and other conditions in Theorem 1 and 2).

(E1) The system (1) is $\mathcal{K} \mathcal{L}$-stable.

(E2) For (1), there exists a LF, $V(x)$ that satisfies (5) and the decrescent condition (6).

(E3) The linearization of (1) given by $\dot{x}=D f(0) x$ is globally exponentially stable and there exists a LF and a norm $\|\cdot\|, V(x)=\|x\|$ that certifies it.

See [18] or any other standard textbook on Lyapunov theory for proofs. Similar to the results for the classical Lyapunov theory, we have the analogous results for finite-time Lyapunov functions and the $\mathcal{K} \mathcal{L}$-stability of system (1) that we can now summarize using all of the results above in the following theorem.

\section{Theorem 5. The following statements are equivalent}

(F1) The system (1) is $\mathcal{K} \mathcal{L}$-stable.

(F2) There exists a d-invariant set $S$ for which $V(x)=\|x\|$ is a FTLF for system (1).

(F3) For the linearization of (1) given by $\dot{x}=A x$, there exists a global FTLF, $V(x)=\|x\|$ and $d>0$ as given in Definition 4.

(F4) There exists a $L F W(x)$ for system (1) that satisfies equations (5) and (6) given by equation (14).

Proof. (F1) implies (F2) from Theorem 4 and (F2) implies (F1) from [10, Theorem 2.1]. (F3) implies (F2) from Theorem 3. The proof for Theorem 4 also gives us that (F1) implies (F3). Hence, (F1), (F2), and (F3) are equivalent. Finally, (F1) and (F4) are equivalent and (F3) implies (F4) by Theorems 1, 2, and 3 respectively hence proving the theorem.

Clearly, from the above results we can also see that all statements (E1)-(E3) and (F1)-(F4) are all equivalent. Note that the equivalence between (E2) and (F2) can also be proven using the approach in [22, Theorem 11].

\section{DOA COMPUTATION}

We begin by discussing a constructive method that can give an estimate of the domain of attraction (DOA) based on direct linearization of the system dynamics around the equilibrium points.

\section{A. DOA estimation using linearization}

Using the continuous-time Lyapunov equation for the linearized dynamics $(A=D f(0)), A^{T} P+P A=-Q$, $P=P^{T} \succ 0, Q=Q^{T} \succ 0$, we can always constructively find a $\operatorname{LF} V(x)=x^{T} P x$. From the classical converse Lyapunov theorem, we have that the same LF is valid locally for the nonlinear dynamics as well. Hence, using the decomposition from equation (15), we can write

$$
\dot{V} \leq-x^{T} Q x+2 x^{T} P g
$$

for the time-derivative of the LF. Using equation (19) and the Cauchy-Schwarz inequality, we can write

$$
x^{T} P g(x) \leq N \lambda_{\max }(P)\|x\|^{2} .
$$


Substituting, we get

$$
\dot{V}(x) \leq-\left(\lambda_{\min }(Q)-2 N \lambda_{\max }(P)\right)\|x\|^{2},
$$

for all $\|x\|<r$. To ensure that $\dot{V}$ is negative, we get the following estimate for the DOA,

$$
N=\max _{\|x\|<r}\left\|\int_{0}^{1}(D(f(\tau x)-D f(0)) x) d \tau\right\|<\frac{\lambda_{\min }(Q)}{2 \lambda_{\max }(P)},
$$

i.e. we can solve the above inequality to find an estimate for $r$ for $x \in \mathcal{B}_{r}(0)$. Therefore, $\mathcal{B}_{r}(0)$ is an estimate of the DOA. As expected, this method gives conservative DOA estimates. We will demonstrate this fact for an example in the next section. On the other hand, there are various other methods that give non-conservative estimates of the DOA that depend on solving an optimization problem. Hence, algorithms based on these methods tend to be computationally inefficient even for simple system dynamics since the validity regions of the LF are computed a posteriori to the construction of the LF. Our method aims to provide the best of both worlds i.e. it provides a constructive approach to compute nonconservative DOA estimates while being computationally efficient.

\section{B. DOA estimates using FTLF theory}

Since the LF derivation given by FTLFs presented in the previous section is a constructive approach, it can be efficiently used for DOA computations for general nonlinear systems. Using the results from Section III, we can construct a simple computational algorithm to compute an estimate for the DOA. This procedure is similar to the algorithm given in [10], however, using the new results that we proved in this paper in Theorems 4 and 5, the DOA estimation algorithm can be presented in a simpler way. In addition, we augment this algorithm by combining a numerical approximation based method to find larger DOA regions. To that end, we will present the algorithm and also briefly allude to how the DOA estimates compare with those obtained using direct linearization.

In general, to find a DOA estimate the following optimization problem needs to be solved for a given $C$ and a $\operatorname{LF} W(x)$,

$$
\begin{aligned}
\max _{x \in S} & \nabla^{T} W(x) f(x) \\
\text { s.t. } & W(x) \leq C
\end{aligned}
$$

The maximum value of $C$ obtained gives us the best estimate for the DOA. We can see that here the Lyapunov level-set $\Omega_{C}=\left\{x \in \mathbb{R}^{n}: W(x) \leq C\right\}$ represents an estimate for the DOA. It is clear that solving the above optimization problem is hard for general nonlinear systems. However, as suggested in [10], we only need to study the feasibility problem of ensuring $W(x) \leq C$ while $\dot{W}$ is negative. We can use the constructive LF derivation given in Theorem 5 to greatly simplify the computations.

From Theorem 4, for a given equilibrium point that is $\mathcal{K} \mathcal{L}$-stable, we know that there always exists a $d$ such that the linearization has a global FTLF. We will use this fact to present a simplified version of the computational procedure given in [10] to find the DOA estimate. In the first step, we begin by computing the linearization of the nonlinear dynamics around the equilibrium point under consideration. For the linearization, we know that since the equilibrium point is $\mathcal{K} \mathcal{L}$-stable, we have

$$
\left\|e^{d A}\right\|<1 \text {. }
$$

We know that such a $d$ exists from Theorem 4 . We also know that $V(x)=\|x\|$ is the global FTLF for the linearization and from Theorem 5, we know that we have a LF for (1) given by

$$
W(x)=\int_{0}^{d} V(x+\tau f(x)) d \tau .
$$

As the final step, we can either solve the optimization

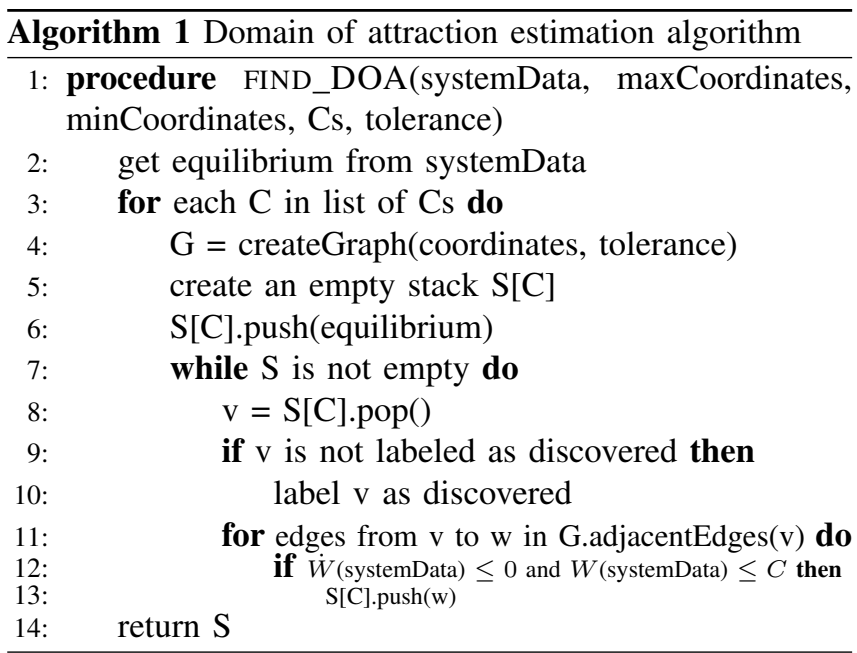

problem given in equation (28) or we could use a numerical approximation algorithm to solve the feasibility problem. The reader is referred to the methods in [23] for expansion methods that could be augmented to this method to improve the DOA estimates further. To solve the feasibility problem, we start with a given value for $C$ and check to see if $\dot{W}$ is negative and proceed in this way until we find the largest value of $C$ with $\dot{W}$ negative. This value for $C$ gives us the DOA estimate as the Lyapunov level set $\Omega_{C}$ around the equilibrium point considered. Since our goal is to find the largest $C$, we can perform a search over the state-space while ensuring that $\dot{W}$ is negative. We present a depth-first search based algorithm (adapted from [25, p. 93]) that finds the best DOA estimate for a given nonlinear system in Algorithm 1.

\section{Toggle Switch}

A toggle switch is a bistable system that can be engineered out of synthetic biological parts. Since the first demonstration of the toggle switch [26] in 2000, there have been various studies and experiments in the past decade that involve the use of such dynamics [27]. We will use the methods given in the previous section to compute an estimate 

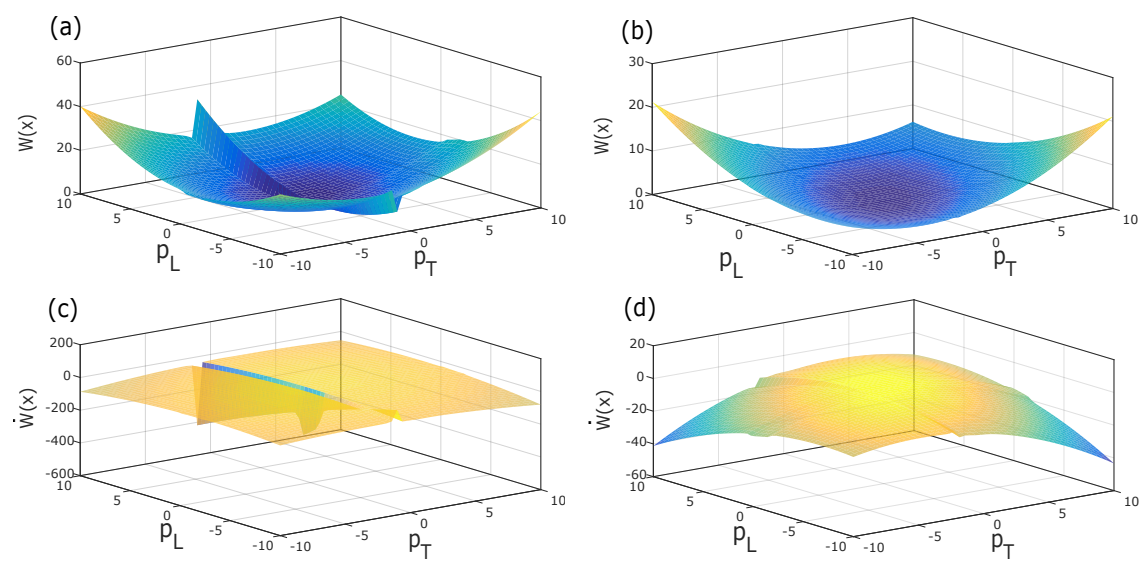

Fig. 1. The figure shows analytically computed LFs, $W(x)$, and their derivatives, $\dot{W}(x)$, for each asymptotically stable equilibrium point $E 2$ and $E 3$ of the toggle switch. The LFs are computed by constructing the global FTLF of the linearized dynamics. The LFs are valid locally for each equilibrium in the $d$-invariant set corresponding to each equilibrium. Our main result in this paper proves the existence of these FTLFs and the LFs for each $\mathcal{K} \mathcal{L}$-stable equilibrium point. (a): $W(x)$ for $E 2$. (b): $W(x)$ for $E 3$. (c): $\dot{W}(x)$ for $E 2$. (d): $\dot{W}(x)$ for $E 3$. The simulation parameters are available at [24].

of the domain of attraction of the stable equilibrium points of the toggle switch dynamics. The system dynamics are described by two protein species, $p_{\mathrm{T}}$ and $p_{\mathrm{L}}$, that repress each other under the presence of corresponding inducer molecules. A reduced order ODE model for this system can be obtained as shown in [28]:

$$
\begin{aligned}
& \dot{p}_{\mathrm{T}}=\frac{K b_{\mathrm{L}}^{n_{\mathrm{L}}}}{b_{\mathrm{L}}^{n_{\mathrm{L}}}+p_{\mathrm{L}}^{n_{\mathrm{L}}}}-d_{\mathrm{T}} p_{\mathrm{T}} \\
& \dot{p}_{\mathrm{L}}=\frac{K b_{\mathrm{T}}^{n_{\mathrm{T}}}}{b_{\mathrm{T}}^{n_{\mathrm{T}}}+p_{\mathrm{T}}^{n_{\mathrm{T}}}}-d_{\mathrm{L}} p_{\mathrm{L}}
\end{aligned}
$$

where $n_{\mathrm{T}}$ and $n_{\mathrm{L}}$ are cooperativity parameters for the repression mechanism [29], $b_{\mathrm{T}}, b_{\mathrm{L}}$ are corresponding activation parameters for the Hill functions, and $d_{\mathrm{T}}, d_{\mathrm{L}}$ are the protein degradation parameters. It has been shown that this system displays bistability properties [29] for $n_{\mathrm{T}}, n_{\mathrm{L}} \geq 2$. It has three equilbirium points out of which two are stable and one

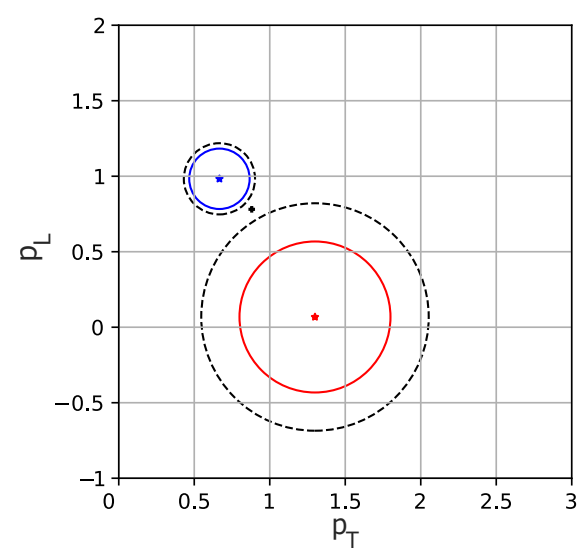

Fig. 2. The domain of attraction estimates obtained using direct linearization based LF construction for the stable equilbrium points (red : E2, blue : E3). The dashed points denote the $d$-invariant sets chosen for each equilibrium point. is unstable. We can compute the equilibrium points and the linearization of the dynamics around these points for DOA estimation. It is important to note that even for this simple system dynamics, directly solving the optimization (28) is a hard problem that cannot be solved by standard convex optimization solvers due to the non-convex nature of the system dynamics. In contrast, our approach that uses FTLFs to construct a LF analytically is computationally efficient to compute non-conservative estimates of the DOA. From the results in [10], we have an estimate for the $d$-invariant set $S \subset\left\{x \in \mathbb{R}^{n}:\|x\| \leq r\right\}$ using the upper bound on $N$ (as given in equation 27)

$$
N \leq \frac{d \mu(A)^{2}}{\zeta} .
$$

For a chosen set of parameters [24], we substitute the values for $d, \zeta$, and $\mu(A)$ to obtain the upper bounds on $N$ for each equilibrium point. Using this bound, for the equilibrium point $E 2$, we choose $r=0.75$ and for $E 3, r=0.425$ to get the region for the $d$-invariant set. The $d$-invariant set estimates are show in Fig. 2 alongside the DOA estimates based on the linearization method (as discussed in Section IV-A) for comparison. Clearly, the linearization based results are conservative estimates since the LF obtained for the linearization is only valid locally in a small region for the nonlinear system. Next, we will use the FTLFs developed for the $d$-invariant set $S$ and the LF derivation from it as described in Section IV-B. We choose the FTLF candidate $V(x)=x^{T} P x$ for each equilbrium point and compute analytically the expression for the LF $W(x)$ using equation (14). For this case, we can also compute analytically the expression for $\dot{W}(x)$. The plots for the LF $W(x)$ and its derivative for each equilibrium point are shown in Fig. 1. Now, by running Algorithm 1, we obtain the domain of attraction estimates that are shown in Fig. 3. Observe that the DOA estimates obtained using our 


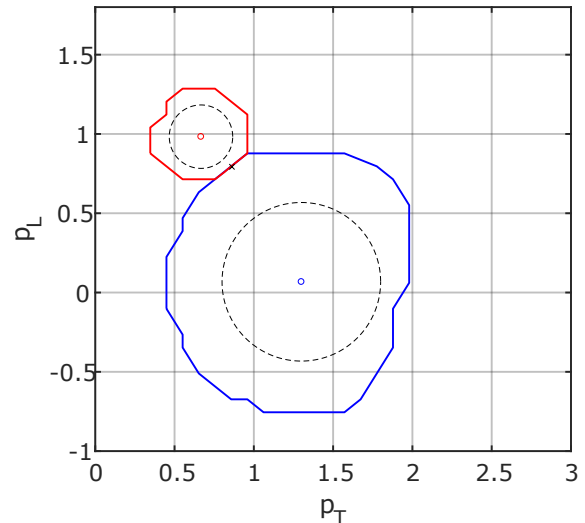

Fig. 3. Comparison of estimates of domain of attractions for the two state toggle switch system. The solid lines are the DOA estimates using the computational procedure discussed in Section IV-B for each equilibrium point. The dashed plots are the DOA estimates obtained by direct linearization. Observe that the boundary of regions for both equilibrium points intersect at the unstable equilibrium point (marked by $\times$ ) suggesting that bigger estimates using this LF might include the unstable point and hence would not be correct DOA estimates.

algorithm are non-conservative and much better estimates than what we get using the linearization method.

\section{CONCLusions}

Our main result in this paper proved a new alternative converse theorem for FTLFs in Theorem 4. Using this result, we stated a consolidated theory for FTLFs in Theorem 5. The implication of the results are significant in simplifying the computation of domain of attraction using FTLFs. In Theorem 4, we show that given asymptotic stability of the equilibrium point of a continuous-time nonlinear dynamical system, there exists a global FTLF for the linearized dynamics. Combining this result with the results in [10] we developed a constructive approach to compute LFs for a nonlinear system that can be efficiently used in DOA computations. We presented a DOA estimation algorithm for general nonlinear systems that uses the results in this paper and combines a numerical depth-first search based algorithm. For a bistable toggle switch dynamics, we demonstrated our results and showed that our algorithm gives non-conservative estimates for the domain of attraction that are much better than the estimates obtained using a direct linearization based method. For future work on these lines, it would be interesting to look at how FTLFs can be used to provide robust stability guarantees by constructing inputto-state stable FTLFs constructively. Similarly, it would be interesting to study controller design methods using FTLFs.

\section{REFERENCES}

[1] J. L. Massera, "On liapounoff's conditions of stability," Annals of Mathematics, pp. 705-721, 1949.

[2] L. F. C. Alberto and H.-D. Chiang, "Characterization of stability region for general autonomous nonlinear dynamical systems," IEEE transactions on automatic control, vol. 57, no. 6, pp. 1564-1569, 2011.

[3] R. E. Kalman and J. E. Bertram, "Control system analysis and design via the "second method" of lyapunov: Continuous-time systems," Journal of Basic Engineering, vol. 82, no. 2, pp. 371-393, 1960.
[4] C. M. Kellett, "Classical converse theorems in lyapunov's second method," arXiv preprint arXiv:1502.04809, 2015.

[5] Q. Zheng and F. Wu, "Regional stabilisation of polynomial non-linear systems using rational lyapunov functions," International Journal of Control, vol. 82, no. 9, pp. 1605-1615, 2009.

[6] O. Hachicho, "A novel lmi-based optimization algorithm for the guaranteed estimation of the domain of attraction using rational lyapunov functions," Journal of the Franklin Institute, vol. 344, no. 5, pp. 535-552, 2007.

[7] F. Amato, C. Cosentino, and A. Merola, "On the region of attraction of nonlinear quadratic systems," Automatica, vol. 43, no. 12, pp. 21192123, 2007.

[8] R. Yedavalli and Z. Liang, "Reduced conservatism in stability robustness bounds by state transformation," IEEE Transactions on Automatic Control, vol. 31, no. 9, pp. 863-866, 1986.

[9] T. A. Johansen, "Computation of lyapunov functions for smooth nonlinear systems using convex optimization," Automatica, vol. 36, no. 11 , pp. 1617-1626, 2000.

[10] A. I. Doban and M. Lazar, "Computation of lyapunov functions for nonlinear differential equations via a massera-type construction," IEEE Transactions on Automatic Control, vol. 63, no. 5, pp. 12591272, 2017.

[11] D. Aeyels and J. Peuteman, "A new asymptotic stability criterion for nonlinear time-variant differential equations," IEEE Transactions on automatic control, vol. 43, no. 7, pp. 968-971, 1998.

[12] R. Geiselhart, R. H. Gielen, M. Lazar, and F. R. Wirth, "An alternative converse lyapunov theorem for discrete-time systems," Systems \& Control Letters, vol. 70, pp. 49-59, 2014.

[13] M. Lazar, A. Doban, and N. Athanasopoulos, "On stability analysis of discrete-time homogeneous dynamics," in 2013 17th International Conference on System Theory, Control and Computing (ICSTCC). IEEE, 2013, pp. 297-305.

[14] R. V. Bobiti and M. Lazar, "On input-to-state stability analysis of discrete-time systems via finite-time lyapunov functions," IFAC Proceedings Volumes, vol. 47, no. 3, pp. 8623-8628, 2014.

[15] K. S. Narendra and A. M. Annaswamy, "Persistent excitation in adaptive systems," International Journal of Control, vol. 45, no. 1, pp. $127-160,1987$.

[16] H. Li and A. Liu, "Computation of non-monotonic lyapunov functions for continuous-time systems," Communications in Nonlinear Science and Numerical Simulation, vol. 50, pp. 35-50, 2017.

[17] W. M. Haddad and V. Chellaboina, Nonlinear dynamical systems and control: a Lyapunov-based approach. Princeton university press, 2011.

[18] H. K. Khalil and J. W. Grizzle, Nonlinear Systems. Prentice hall Upper Saddle River, NJ, 2002, vol. 3.

[19] G. Söderlind, "The logarithmic norm. history and modern theory," BIT Numerical Mathematics, vol. 46, no. 3, pp. 631-652, 2006.

[20] E. D. Sontag, "Comments on integral variants of iss," Systems \& Control Letters, vol. 34, no. 1-2, pp. 93-100, 1998.

[21] R. Bellman and K. Cooke, "Differential-difference equations, acad," Press, NY, 1963.

[22] H. Li and A. Liu, "Computation of non-monotonic lyapunov functions for continuous-time systems," Communications in Nonlinear Science and Numerical Simulation, vol. 50, pp. 35-50, 2017.

[23] H.-D. Chiang and J. S. Thorp, "Stability regions of nonlinear dynamical systems: A constructive methodology," IEEE Transactions on Automatic Control, vol. 34, no. 12, pp. 1229-1241, 1989.

[24] A. Pandey, "Python based domain of attraction estimation software," Online, accessed September 27, 2019.

[25] J. Kleinberg and E. Tardos, Algorithm design. Pearson Education India, 2006.

[26] T. S. Gardner, C. R. Cantor, and J. J. Collins, "Construction of a genetic toggle switch in escherichia coli," Nature, vol. 403, no. 6767, p. 339, 2000.

[27] J.-B. Lugagne, S. S. Carrillo, M. Kirch, A. Köhler, G. Batt, and P. Hersen, "Balancing a genetic toggle switch by real-time feedback control and periodic forcing," Nature communications, vol. 8, no. 1, p. 1671, 2017.

[28] A. Pandey and R. M. Murray, "An automated model reduction tool to guide the design and analysis of synthetic biological circuits," bioRxiv, p. 640276, 2019.

[29] D. Del Vecchio and R. M. Murray, Biomolecular Feedback Systems. Princeton University Press Princeton, NJ, 2015. 